Article

\title{
Synergistic Antibacterial Effects of Probiotic Lactic Acid Bacteria with Curcuma longa Rhizome Extract as Synbiotic against Cutibacterium acnes
}

\author{
Jina Kim, Hyaekang Kim (D), Soomin Jeon, Jinchul Jo, Younghoon Kim (D) and Heebal Kim * \\ Department of Agricultural Biotechnology and Research Institute of Agriculture and Life Sciences, \\ Seoul National University, Seoul 08826, Korea; jinak750@gmail.com (J.K.); hkim458@snu.ac.kr (H.K.); \\ soty23@snu.ac.kr (S.J.); macjoo2000@snu.ac.kr (J.J.); ykeys2584@snu.ac.kr (Y.K.) \\ * Correspondence: heebal@snu.ac.kr; Tel.: +82-2-880-4822; Fax: +82-2-883-8812
}

Received: 28 October 2020; Accepted: 13 December 2020; Published: 15 December 2020

\begin{abstract}
Acne is a chronic inflammatory skin disease induced by Cutibacterium acnes. Recently, the effects of probiotics, prebiotics and synbiotics have been researched for the treatment of skin diseases in humans. However, the synbiotic effect of probiotics and prebiotic Curcuma longa rhizome extract (CLE) on C. acnes remains ambiguous. Therefore, the aim of this study was to investigate the synergistic antibacterial activities of probiotic lactic acid bacteria (LAB) with CLE as a synbiotic against $C$. acnes. Agar well diffusion assays were performed to determine the inhibitory effects of each combination of one of five Lactobacillus sp. with CLE as synbiotics against C. acnes KCTC 3314. Among them, the comparison between the average diameters of inhibition zones showed that the synbiotic combination of Lactobacillus acidophilus A001F8 and CLE significantly increased the inhibition zone diameters against $C$. acnes, compared to the use of Lactobacillus acidophilus A001F8 or CLE alone $(p<0.05)$. In conclusion, the synbiotics of probiotic LAB and CLE showed synergistic antibacterial effects against $C$. acnes, suggesting therapeutic potential for this synbiotic combination in the development of cosmetics or medicine against $C$. acnes.
\end{abstract}

Keywords: Cutibacterium acnes; antibacterial activity; Curcuma longa; synbiotics; synergy; pre-and probiotics

\section{Introduction}

Acne vulgaris is a common skin disease resulting from sebum production in hair follicles on the face, neck and chest, induced by androgen, altered keratinization, inflammation and bacterial colonization. Among them, Cutibacterium acnes is known as a major cause [1].

Probiotics are live bacteria that have beneficial effects on the host; they have been proposed as an alternative to antibiotics, as the indiscriminate use of the latter can cause multidrug resistance or other side effects [2,3]. The direct antibacterial effects of probiotics against pathogens may be observed by inhibiting the growth of pathogens by the production of antimicrobial peptides and colonization resistance [4]. Therefore, probiotics have potential for use as cosmetic or medicinal ingredients against $C$. acnes.

Prebiotics are defined as nondigestible food ingredients, such as oligosaccharides, inulin and lactulose, which promote the growth and survival of probiotics; they are found in vegetables and fruit $[5,6]$. In human skin, prebiotic substances can be selectively applied to modulate microbial systems to achieve beneficial effects [7]. In order to develop natural prebiotics, various medicinal plants have been investigated. Among them, turmeric (Curcuma longa rhizome) is well known for its anti-inflammatory, antimicrobial and antioxidant activities [8]. A previous study reported that Curcuma longa rhizome extract (CLE) reduced the levels of reactive oxygen species (ROS) and cytokines produced by C. acnes [9]. However, few studies have focused on CLE as a prebiotic, and none has reported synergistic effects of combinations of probiotics and CLE as synbiotics on C. acnes. 
Therefore, in this study, the synergistic effects of probiotics and prebiotics against $C$. acnes were investigated. First, the antibacterial effects on $C$. acnes of five potential probiotic strains were assessed. Then, the extracts of twelve natural medicinal plants that have prebiotic potential were screened for their antibacterial activities against $C$. acnes using antibacterial assays. It was found that only CLE displayed inhibition zones. Finally, each of the five lactic acid bacteria (LAB) species was applied with CLE to examine synergistic antibacterial effects on C. acnes.

\section{Materials and Methods}

\subsection{Preparation of Plant Extracts}

Eleven methanolic plant extracts (Cornus officinalis, Allium cepa, Cudrania tricuspidata, Spinacia oleracea, Rubus coreanus, Daucus carota var. sativa, Chrysanthemum coronarium var. spatiosum, Brassica oleracea var. capitate, Brassica oleracea var. capitate, Mollugo verticillata and Schisandra chinensis) and one ethanolic medicinal plant extract (Curcuma longa L.) rhizome used in this study were purchased from the Korea Plant Extract Bank, Korea Research Institute of Bioscience and Biotechnology (KRIBB; Daejeon, South Korea). All plant extracts were dissolved in dimethylsulfoxide (DMSO; Sigma, St Louis, MO, USA) to $10 \%$ of stock concentration and kept at $-20{ }^{\circ} \mathrm{C}$, and then $0.01 \%$ of test concentration [10] in phosphate buffered saline (PBS) solution was used for antibacterial test.

\subsection{Bacterial Species and Growth Conditions}

The species of probiotic lactic acid bacteria (LAB) used in this study were Lactobacillus acidophilus A001F8, Lactobacillus rhamnosus A001G8, Lactobacillus paracasei A002C5, Lactobacillus plantarum A003A7 and Lactobacillus casei A003D4, all of which were isolated from fermented dairy food. These species were identified by 16Sr RNA gene sequencing. C. acnes KCTC 3314 was obtained from the Korean Collection for Type Cultures (KCTC; Daejeon, South Korea). The Lactobacillus species were grown in De Man, Rogosa, Sharpe broth (MRS broth; Difco, Detroit, MI, USA). C. acnes was grown at $37^{\circ} \mathrm{C}$ for $72 \mathrm{~h}$ in brain heart infusion broth (BHI broth; Difco, Detroit, MI, USA) and BHI agar under anaerobic conditions using GasPak pouch (BD GasPak ${ }^{\mathrm{TM}}$ EZ, Franklin Lakes, NJ, USA).

\subsection{Antibacterial Activity by Agar Well Diffusion Assay}

An agar well diffusion assay was performed to determine the antibacterial effects of the plant extracts, LAB with and without CLE against C. acnes, respectively. Vancomycin supplement for microbiology (Sigma; St Louis, MO, USA) was diluted in distilled water (DW) and used as a positive control. Each of the MRS broths was cultured with LAB with $0.01 \%$ DMSO as a control, and LAB with $0.01 \% \mathrm{CLE}$ at $37^{\circ} \mathrm{C}$ for $24 \mathrm{~h}$. First, the inocula of $\mathrm{C}$. acnes $(150 \mu \mathrm{L})$ were spread using sterile cotton swabs on BHI agar plates. Agar wells $(8 \mathrm{~mm})$ were punched with sterile pipette tips. Then, $100 \mu \mathrm{L}$ plant extracts and LAB with and without $0.01 \%$ CLE were added to the agar well. All plates were incubated for $72 \mathrm{~h}$ at $37^{\circ} \mathrm{C}$ under anaerobic conditions using GasPak (BD GasPak ${ }^{\mathrm{TM}} \mathrm{EZ}$, Franklin Lakes, NJ, USA). The tests were performed in three independent experiments, and results are presented as the average values of the diameters of the inhibition zones [11].

\subsection{Determination of Growth Curves of Probiotics with and without CLE}

Lactobacillus sp. (L. acidophilus A001F8, L. rhamnosus A001G8, L. paracasei A002C5, L. plantarum A003A7 and L. casei A003D4) with and without $0.01 \% \mathrm{CLE}$ were cultured at $37^{\circ} \mathrm{C}$ for $18 \mathrm{~h}$. The absorbance was measured by a UV-spectrophotometer (Optizen POP, Mecasys, Korea) at $600 \mathrm{~nm}$ (OD600) every $2 \mathrm{~h}$ for $18 \mathrm{~h}$. To ensure accuracy, each sample was ten-fold serially diluted with PBS.

\subsection{Statistical Analysis}

All data are shown as mean \pm standard deviation (SD) for the quantitative variables. Frequencies and graphs are given for qualitative variables. A Student's $t$-test was performed using SPSS version 
23 statistics software to determine statistically significant differences between the measurements of $\mathrm{LAB}$ alone and $\mathrm{LAB}+\mathrm{CLE}$. A value of $p<0.05$ was considered statistically significant.

\section{Results}

\subsection{Screening of Antibacterial Activities of Plant Extracts}

An agar well diffusion assay was conducted to assess the antibacterial activities of twelve plant extracts against $C$. acnes. Antibacterial activities were recorded as the average of the diameters of the inhibition zones. As shown in Table 1, vancomycin $(30 \mu \mathrm{g} /$ well $)$, used as a positive control, showed a $13.6 \pm 0.29-\mathrm{mm}$ inhibition zone against $C$. acnes. With the exception of CLE, none of the plant extracts displayed inhibition zones. CLE showed a $10.8 \pm 0.29$-mm inhibition zone. These results indicated that CLE has antibacterial activity.

Table 1. The inhibitory effects of plant extracts against C. acnes.

\begin{tabular}{ccc}
\hline Name of Plant & Extracted Part & $\begin{array}{c}\text { Inhibition Zone } \\
(\mathbf{m m})\end{array}$ \\
\cline { 3 - 3 } & & $\begin{array}{c}\text { Cutibacterium acnes } \\
\text { Cornus officinalis }\end{array}$ \\
Allium cepa & Berry & - \\
Curcuma longa L. & Whole plant & - \\
Cudrania tricuspidata & Rhizome & $10.8 \pm 0.29$ \\
Spinacia oleracea & Root & - \\
Rubus coreanus & Whole plant & - \\
Daucus carota var. sativa & Leaves, Stem & - \\
Chrysanthemum coronarium var. spatiosum & Whole plant & - \\
Brassica oleracea var. capitata & Whole plant & - \\
Zingiber officinale & Whole plant & - \\
Mollugo verticillata & Root & - \\
Schisandra chinensis & Whole plant & - \\
Vancomycin (Positive control) & Leaves & - \\
\hline
\end{tabular}

\subsection{Synergistic Antibacterial Effects of Lactobacillus sp. with and without CLE against C. acnes}

As shown in Figure 1, the antibacterial properties against $C$. acnes of each LAB species alone and with CLE were compared on five sets using agar well diffusion assay. Among them, L. acidophilus A001F8 showed the highest synergetic antibacterial activity, and the average value of the inhibition zone diameters of L. acidophilus A001F8 + CLE $(13.8 \pm 0.29)$ was significantly increased compared to L. acidophilus A001F8 alone $(12.3 \pm 0.57)(p<0.05)$. Although for other LAB (L. rhamnosus A001G8, L. paracasei $\mathrm{A} 002 \mathrm{C} 5$, L. plantarum A003A7 and L. casei A003D4) there was no statistically significant difference, the average values of the inhibition zones of $L A B+C L E$ were larger than those of $L A B$ alone.

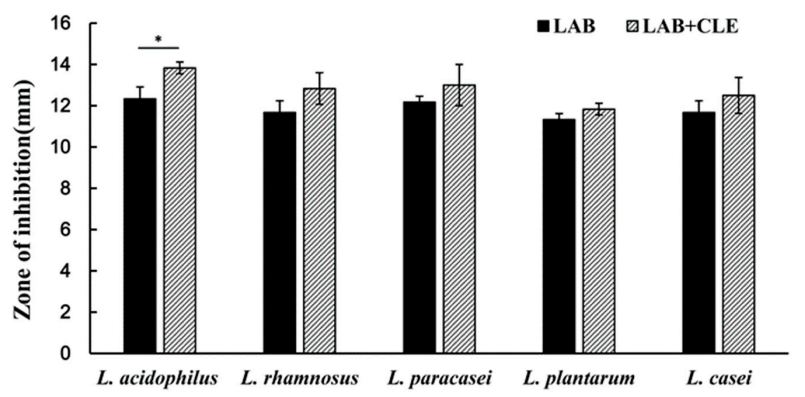

Figure 1. The synergistic antibacterial effects of Lactobacillus sp. with and without CLE against C. acnes. Data were analyzed by Student's $t$-test and expressed as the means \pm SD of three independent experiments $\left({ }^{*} p<0.05\right)$. 


\subsection{Effect of CLE on the Growth of Five Lactobacillus sp.}

The growth curves of five Lactobacillus sp. (L. acidophilus A001F8, L. rhamnosus A001G8, L. paracasei A002C5, L. plantarum A003A7 and L. casei A003D4) with and without CLE showed no significant differences for each set, implying that CLE did not stimulate growth of Lactobacillus sp. (Figure 2).
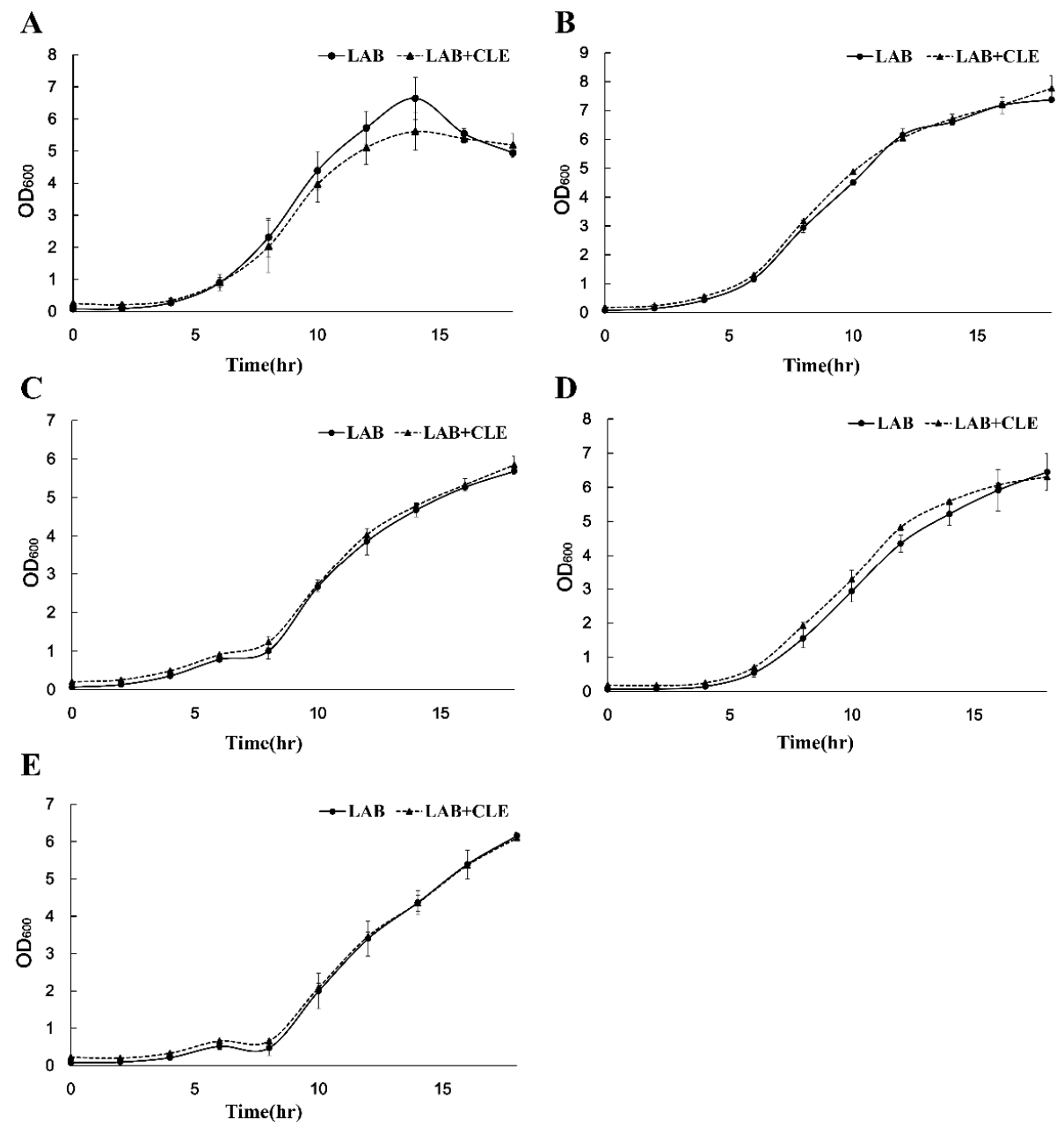

Figure 2. The growth curve of L. acidophilus A001F8 (A), L. rhamnosus A001G8 (B), L. paracasei A002C5 (C), L. plantarum A003A7 (D) and L. casei A003D4 (E), with and without CLE. There were no statistically significant differences in growth between LAB and LAB + CLE (Student's $t$-test; $p>0.05$ ).

\section{Discussion}

Probiotics have the potential to prevent infections from pathogenic bacteria and promote the colonization of a healthy microbiome, thereby serving as potential alternative dermatological treatments [12]. Moreover, the use of synbiotics, i.e., combinations of probiotics and prebiotics, has been proposed to treat several skin conditions such as atopic dermatitis, acne, rosacea and wounds [11,13]. In this study, the synergistic antibacterial effects of probiotic LAB with CLE were evaluated against C. acnes using the same agar well diffusion assay applied in previous experiments [11,14]. First, a total of twelve candidate plant extracts that have prebiotic potential were selected based on a literature review $[6,15]$. These extracts were then subjected to screening for their antibacterial activities against C. acnes. The CLE was finally selected as the most effective substance in terms of its synergistic effect with probiotics to inhibit the growth of $C$. acnes.

Five combinations of LAB and CLE displayed more positive synergistic effects against the growth of $C$. acnes than LAB or CLE alone in the agar well diffusion assay. In this study, there were no 
significant differences in the growth activities of five Lactobacillus sp. when CLE was added at a relatively low concentration of $0.01 \%$, but a recent study reported that a $2 \%$ turmeric extract promoted the growth of L. rhamnosus GG (LGG) and Bifidobacterium animalis BB12 [16]. The use of different strains or concentrations of CLE could be the cause of the disagreement between our results and previous data.

According to previous studies, LAB are capable of inhibiting pathogen growth through the antibiofilm effects of biosurfactants or the antimicrobial effects of substances like bacteriocin $[17,18]$. Also, the bacteriocin produced by Lactococcus sp. HY 449 was reported to have a bacteriolytic action on the cell wall and membrane of C. acnes [19]. Moreover, prebiotics such as oligosaccharides (OS), inulin and lactulose are known to enhance the secretion and activity of bacteriocin produced by LAB $[20,21]$. Although the mechanisms of the enhancing action remain obscure, our results presented a novel synergistic anti-C. acnes effect of CLE in combination with probiotic LAB. Future studies are needed to investigate the metabolites produced by the synbiotics. Based on the above findings, cosmetics containing a combination of L. acidophilus A001F8 with CLE could have the potential to alleviate acne. Moreover, the systemic supplementation with these synbiotics could a positive effect on acne, according to a previous study [22].

In conclusion, these results suggest that $L$. acidophilus A001F8 with prebiotic CLE has a synergistic effect of antibacterial activity against $C$. acnes, and thus, that prebiotic CLE can be utilized for the development of cosmetics and medicines for the treatment of acne.

Author Contributions: Conceptualization, J.K. and H.K. (Heebal Kim); Investigation, J.K., H.K. (Hyaekang Kim); methodology, J.K., S.J., H.K. (Hyaekang Kim); formal analysis, S.J., J.J.; writing—original draft preparation, J.K.; writing—review and editing, H.K. (Hyaekang Kim), Y.K., H.K. (Heebal Kim); Supervision, H.K. (Heebal Kim). All authors have read and agreed to the published version of the manuscript.

Funding: This research received no external funding.

Conflicts of Interest: The authors declare no conflict of interest.

\section{References}

1. Gribbon, E.; Cunliffe, W.; Holland, K. Interaction of Propionibacterium acnes with skin lipids in vitro. Microbiology 1993, 139, 1745-1751. [CrossRef] [PubMed]

2. Salminen, S.; Ouwehand, A.; Benno, Y.; Lee, Y. Probiotics: How should they be defined? Trends Food Sci. Technol. 1999, 10, 107-110. [CrossRef]

3. Mottin, V.H.; Suyenaga, E.S. An approach on the potential use of probiotics in the treatment of skin conditions: Acne and atopic dermatitis. Int. J. Dermatol. 2018, 57, 1425-1432. [CrossRef] [PubMed]

4. Nair, M.S.; Amalaradjou, M.; Venkitanarayanan, K. Antivirulence Properties of Probiotics in Combating Microbial Pathogenesis. In Advances in Applied Microbiology; Academic Press: Cambridge, MA, USA, 2017; Volume 98, pp. 1-29.

5. Gibson, G.R.; Roberfroid, M.B. Dietary modulation of the human colonic microbiota: Introducing the concept of prebiotics. J. Nutr. 1995, 125, 1401-1412. [CrossRef]

6. Al-Sheraji, S.H.; Ismail, A.; Manap, M.Y.; Mustafa, S.; Yusof, R.M.; Hassan, F.A. Prebiotics as functional foods: A review. J. Funct. Foods 2013, 5, 1542-1553. [CrossRef]

7. Krutmann, J. Pre-and probiotics for human skin. J. Dermatol. Sci. 2009, 54, 1-5. [CrossRef]

8. Vaughn, A.R.; Branum, A.; Sivamani, R.K. Effects of turmeric (Curcuma longa) on skin health: A systematic review of the clinical evidence. Phytother. Res. 2016, 30, 1243-1264. [CrossRef]

9. Jain, A.; Basal, E. Inhibition of Propionibacterium acnes-induced mediators of inflammation by Indian herbs. Phytomedicine 2003, 10, 34-38. [CrossRef]

10. Nam, C.; Kim, S.; Sim, Y.; Chang, I. Anti-acne effects of Oriental herb extracts: A novel screening method to select anti-acne agents. Ski. Pharmacol. Physiol. 2003, 16, 84-90. [CrossRef]

11. Al-Ghazzewi, F.H.; Tester, R. Effect of konjac glucomannan hydrolysates and probiotics on the growth of the skin bacterium Propionibacterium acnes in vitro. Int. J. Cosmet. Sci. 2010, 32, 139-142. [CrossRef]

12. Yu, Y.; Dunaway, S.; Champer, J.; Kim, J.; Alikhan, A. Changing our microbiome: Probiotics in dermatology. Br. J. Dermatol. 2020, 182, 39-46. [CrossRef] 
13. Kearney, S.M.; Gibbons, S.M. Designing synbiotics for improved human health. Microb. Biotechnol. 2018, 11, 141-144. [CrossRef] [PubMed]

14. Vora, J.; Srivastava, A.; Modi, H. Antibacterial and antioxidant strategies for acne treatment through plant extracts. Inform. Med. Unlocked 2018, 13, 128-132. [CrossRef]

15. Holscher, H.D. Dietary fiber and prebiotics and the gastrointestinal microbiota. Gut Microbes 2017, 8, 172-184. [CrossRef] [PubMed]

16. Yazdi, F.G.; Soleimanian-Zad, S.; van den Worm, E.; Folkerts, G. Turmeric Extract: Potential Use as a Prebiotic and Anti-Inflammatory Compound? Plant Foods Hum. Nutr. 2019, 74, 293-299. [CrossRef] [PubMed]

17. Khalaf, H.; Nakka, S.S.; Sandén, C.; Svärd, A.; Hultenby, K.; Scherbak, N.; Aili, D.; Bengtsson, T. Antibacterial effects of Lactobacillus and bacteriocin PLNC8 $\alpha \beta$ on the periodontal pathogen Porphyromonas gingivalis. BMC Microbiol. 2016, 16, 188. [CrossRef] [PubMed]

18. Yan, X.; Gu, S.; Cui, X.; Shi, Y.; Wen, S.; Chen, H.; Ge, J. Antimicrobial, anti-adhesive and anti-biofilm potential of biosurfactants isolated from Pediococcus acidilactici and Lactobacillus plantarum against Staphylococcus aureus CMCC26003. Microb. Pathog. 2019, 127, 12-20. [CrossRef]

19. Oh, S.; Kim, S.-H.; Ko, Y.; Sim, J.-H.; Kim, K.S.; Lee, S.-H.; Park, S.; Kim, Y.J. Effect of bacteriocin produced by Lactococcus sp. HY 449 on skin-inflammatory bacteria. Food Chem. Toxicol. 2006, 44, 552-559. [CrossRef]

20. Pranckutè, R.; Kaunietis, A.; Kuisienè, N.; Čitavičius, D.J. Combining prebiotics with probiotic bacteria can enhance bacterial growth and secretion of bacteriocins. Int. J. Biol. Macromol. 2016, 89, 669-676. [CrossRef]

21. Chen, Y.S.; Srionnual, S.; Onda, T.; Yanagida, F. Effects of prebiotic oligosaccharides and trehalose on growth and production of bacteriocins by lactic acid bacteria. Lett. Appl. Microbiol. 2007, 45, 190-193. [CrossRef]

22. Fabbrocini, G.; Bertona, M.; Picazo, O.; Pareja-Galeano, H.; Monfrecola, G.; Emanuele, E. Supplementation with Lactobacillus rhamnosus SP1 normalises skin expression of genes implicated in insulin signalling and improves adult acne. Benef. Microbes 2016, 7, 625-630. [CrossRef] [PubMed]

Publisher's Note: MDPI stays neutral with regard to jurisdictional claims in published maps and institutional affiliations.

(C) 2020 by the authors. Licensee MDPI, Basel, Switzerland. This article is an open access article distributed under the terms and conditions of the Creative Commons Attribution (CC BY) license (http://creativecommons.org/licenses/by/4.0/). 